\title{
THE ZERO-DIVISOR GRAPH UNDER GROUP ACTIONS IN A NONCOMMUTATIVE RING
}

\author{
JUNCHEOL HAN
}

\begin{abstract}
Let $R$ be a ring with identity, $X$ the set of all nonzero, nonunits of $R$ and $G$ the group of all units of $R$. First, we investigate some connected conditions of the zero-divisor graph $\Gamma(R)$ of a noncommutative ring $R$ as follows: (1) if $\Gamma(R)$ has no sources and no sinks, then $\Gamma(R)$ is connected and diameter of $\Gamma(R)$, denoted by $\operatorname{diam}(\Gamma(R))$ (resp. girth of $\Gamma(R)$, denoted by $g(\Gamma(R)))$ is equal to or less than 3; (2) if $X$ is a union of finite number of orbits under the left (resp. right) regular action on $X$ by $G$, then $\Gamma(R)$ is connected and $\operatorname{diam}(\Gamma(R))$ (resp. $g(\Gamma(R)))$ is equal to or less than 3 , in addition, if $R$ is local, then there is a vertex of $\Gamma(R)$ which is adjacent to every other vertices in $\Gamma(R)$; (3) if $R$ is unitregular, then $\Gamma(R)$ is connected and $\operatorname{diam}(\Gamma(R))$ (resp. $g(\Gamma(R)))$ is equal to or less than 3. Next, we investigate the graph automorphisms group of $\Gamma\left(\operatorname{Mat}_{2}\left(\mathbb{Z}_{p}\right)\right)$ where $\operatorname{Mat}_{2}\left(\mathbb{Z}_{p}\right)$ is the ring of 2 by 2 matrices over the galois field $\mathbb{Z}_{p}$ ( $p$ is any prime).
\end{abstract}

\section{Introduction and basic definitions}

The zero-divisor graph of a commutative ring has been studied extensitively by Akbari, Anderson, Frazier, Lauve, Livinston, and Maimani in [1, 2, 3] since its concept had been introduced by Beck in [4]. Recently, the zero-divisor graph of a noncommutative ring (resp. a semigroup) has also been studied by Redmond and Wu (resp. F. DeMeyer and L. DeMeyer) in [12, 13, 14] (resp. $[6]$ ). The zero-divisor graph is very useful to find the algebraic structures and properties of rings. In this paper, the zero-divisor graph of a noncommutative ring is also studied by considering some group actions.

Throughout this paper all rings are assumed to be rings with identity. For a ring $R$, let $Z_{\ell}(R)$ (resp. $Z_{r}(R)$ ) be the set of all left (resp. right) zerodivisors of $R, Z(R)=Z_{\ell}(R) \cup Z_{r}(R)$ and $\Gamma(R)$ be the zero-divisor graph of $R$ consisting of all vertices in $Z(R)^{*}=Z(R) \backslash\{0\}$, the set of all nonzero left or right zero-divisors of $R$, and edges $x \longrightarrow y$, which means that $x y=0$ for $x, y \in Z(R)^{*}$. If there exist vertices $x_{0}, \ldots, x_{n} \in Z(R)^{*}$ such that $P$ :

Received March 7, 2007; Revised October 4, 2007.

2000 Mathematics Subject Classification. Primary 05C20; Secondary 16W22.

Key words and phrases. connected (resp. complete) zero-divisor graph, left (resp. right) regular action, orbit, graph automorphisms group. 
$x_{0} \longrightarrow x_{1} \longrightarrow \cdots \longrightarrow x_{n-1} \longrightarrow x_{n}$ where $x_{i} \neq x_{j}$ for all $i, j=0,1, \ldots, n$ $(i \neq j)$ for some positive integer $n$, then $P$ is called a path from $x_{0}$ to $x_{n}$ of length $n$. We will denote $d(x, y)$ by the length of the shortest path from $x$ to $y$, otherwise, $d(x, y)=\infty$. Recall that $\Gamma(R)$ is connected if for all distinct vertices $x, y \in Z(R)^{*}$ there exists a path from $x$ to $y$. The diameter of $\Gamma(R)$ (denoted by $\operatorname{diam}(\Gamma(R)))$ is defined by the supremum of $d(x, y)$ for all distinct vertices $x$ and $y$ in $\Gamma(R)$. In particular, if $x=y$ and $d(x, x)=k$, then the path is called the cycle of length $k$. Usually vertices of a path may be considered to be distinct, however in a cycle, the initial and the final vertices are the same. If $\Gamma(R)$ contains a cycle, then the girth of $\Gamma(R)$ (denoted by $g(\Gamma(R))$ ) is defined by the length of the shortest cycle in $\Gamma(R)$, otherwise, $g(\Gamma(R))=\infty$. In $[7$, Proposition 1.3.2], if $\Gamma(R)$ contains a cycle, then $1+2 \operatorname{diam}(\Gamma(R)) \geq g(\Gamma(R))$. We say that $\Gamma(R)$ is complete if $x y=0$ for any distinct vertices $x, y$ in $\Gamma(R)$.

For a ring $R$, let $X(R)$ (simply, denoted by $X$ ) be the set of all nonzero, nonunits of $R, G(R)$ (simply, denoted by $G$ ) be the group of all units of $R$ and $J(R)$ (simply, denoted by $J$ ) be the Jacobson radical of $R$. In this paper, we will consider some group actions on $X$ by $G$ given by $(g, x) \longrightarrow g x$ (resp. $\left.(g, x) \longrightarrow x g^{-1}\right)$ from $G \times X$ to $X$, called the left (resp. right) regular action. If $\phi: G \times X \longrightarrow X$ is the left (resp. right) regular action, then for each $x \in X$, we define the orbit of $x$ by $o_{\ell}(x)=\{\phi(g, x)=g x: \forall g \in G\}$ (resp. $o_{r}(x)=\left\{\phi(g, x)=x g^{-1}: \forall g \in G\right\}$ ). Recall that $G$ is transitive on $X$ (or $G$ acts transitively on $X$ ) under the regular action on $X$ by $G$ if there is an $x \in X$ with $o_{\ell}(x)=X$ (resp. $o_{r}(x)=X$ ) and the left (resp. right) regular action on $X$ by $G$ is trivial if $o_{\ell}(x)=\{x\}$ (resp. $\left.o_{r}(x)=\{x\}\right)$ for all $x \in X$. In [8], it has been shown that if $X$ is a union of a finite $n$ number of orbits under the left regular action on $X$ by $G$, then $x^{n+1}=0$ for all $x \in J$ and $X$ is the set of all nonzero right zero-divisors of $R$. Similarly, it is also shown that if $X$ is a union of a finite $n$ number of orbits under the right regular action on $X$ by $G$, then $x^{n+1}=0$ for all $x \in J$ and $X$ is the set of all nonzero left zero-divisors of $R$.

Recall that for all $x \in X$ the set $\operatorname{ann}_{\ell}(x)=\{y \in X: y x=0\}$ (resp. $\operatorname{ann}_{r}(x)=\{z \in X: x z=0\}$ ) is called a left (resp. right) annihilator of $x$. Let $\operatorname{ann}_{\ell}^{*}(x)=\operatorname{ann}_{\ell}(x) \backslash\{0\}\left(\operatorname{resp} . \operatorname{ann}_{r}^{*}(x)=\operatorname{ann}_{r}(x) \backslash\{0\}\right)$. Given a zerodivisor graph $\Gamma(R)$ and a vertex $x \in Z(R)^{*}$, the indegree (resp. outdegree) of $x$ (denoted by indegree $(x)$ (resp. outdegree $(x)$ ) is the number of edges arriving (resp. leaving) at $x$. That is, indegree $(x)=\left|\operatorname{ann}_{\ell}^{*}(x)\right|$ (resp. outdegree $(x)=$ $\left.\left|\operatorname{ann}_{r}^{*}(x)\right|\right)$. A vertex of indegree 0 (resp. outdegree 0 ) is called a source (resp. $\operatorname{sink})$.

In Section 2, some connected conditions of the zero-divisor graph of a noncommutative ring $R$ are investigated as follows: (1) if $\Gamma(R)$ has no sources and no sinks, then $\Gamma(R)$ is connected and $\operatorname{diam}(\Gamma(R))$ (resp. $g(\Gamma(R)))$ is equal to or less than $3 ;(2)$ if $X$ is a union of finite number of orbits under the left (resp. right) regular action on $X$ by $G$, then $\Gamma(R)$ is connected and $\operatorname{diam}(\Gamma(R))$ (resp. $g(\Gamma(R))$ ) is equal to or less than 3 , in addition, if $R$ is a local ring, then there exists a vertex of $\Gamma(R)$ which is adjacent to every other vertices in $\Gamma(R)$; 
(4) if $R$ is a unit-regular ring, then $\Gamma(R)$ is connected and $\operatorname{diam}(\Gamma(R))$ (resp. $g(\Gamma(R)))$ is equal to or less than 3 .

In [3], Anderson and Livingston have shown that distinct ring automorphisms of a finite ring $R$ which is not a field induce distinct graph automorphisms of $\Gamma(R)$ and determined $\operatorname{Aut}(\Gamma(R))$, the graph automorphisms group of $\Gamma(R)$. In particular, they have computed $\operatorname{Aut}\left(\Gamma\left(\mathbb{Z}_{n}\right)\right)$.

In Section 3, when $R=\operatorname{Mat}_{2}\left(\mathbb{Z}_{p}\right)$, the ring of 2 by 2 matrices over the Galois field $\mathbb{Z}_{p}$ ( $p$ is any prime), we will show that $\operatorname{Aut}(\Gamma(R))$ is isomorphic to the group $S_{p+1}$, the symmetric group of degree $p+1$ by investigating that (1) the number of orbits under the left (resp. right) regular action on $X$ by $G$ is $p+1$; (2) the number of nonzero nilpotents in $R$ is $p^{2}-1$; (3) $\operatorname{Aut}(\Gamma(R)) \neq\{1\}$; (4) under the left (resp. right) regular action on $X$ by $G, o_{\ell}(a) \cap N(p)=o_{r}(a) \cap N(p)=$ $o_{\ell}(a) \cap o_{r}(a)$ for all $a \in N(p)$ where $N(p)$ is the set of all nonzero nilpotents in $R$.

\section{Connected zero-divisor graph under the left (resp. right) regular action}

For a subset $S$ of $Z(R)^{*}$, we will denote the subgraph of $\Gamma(R)$ with vertices in $S$ by $\Gamma_{S}(R)$.

Proposition 2.1. Let $R$ be a ring. If the left (or right) regular action of $G$ on $X$ is transitive, then $\Gamma_{X}(R)$ is complete.

Proof. Since the left regular action of $G$ on $X$ is transitive, $R$ is a local ring and $J^{2}=0$ by [8, Corollary 2.4], and so $Z(R)^{*}=X$ and $\Gamma_{X}(R)$ is complete. If the right regular action of $G$ on $X$ is transitive, then $Z(R)^{*}=X$ and $\Gamma_{X}(R)$ is also complete by the similar argument.

Remark 1. In Proposition 2.1, we see that if the left (resp. right) regular action on $X$ by $G$ is transitive, then $x^{2}=0$, i.e., $x$ is a nilpotent element of nilpotency 2 for all $x \in X$.

Theorem 2.2. Let $R$ be a ring. If $\Gamma(R)$ has no sources and no sinks, then $\Gamma(R)$ is connected and $\operatorname{diam}(\Gamma(R))$ (resp. $g(\Gamma(R))$ ) is equal to or less than 3.

Proof. Let $x, y \in Z(R)^{*}(x \neq y)$ be arbitrary. Since $\Gamma(R)$ has no sources and no sinks, i.e., $\operatorname{ann}_{\ell}^{*}(x) \neq \emptyset$ (resp. $\operatorname{ann}_{r}^{*}(x) \neq \emptyset$ ), there exists an element $a \in X$ (resp. $b \in X$ ) such that $x a=0$ (resp. $b y=0$ ). If $a b=0$, then $x \longrightarrow a \longrightarrow$ $b \longrightarrow y$ is a path of length 3 . If $a b \neq 0$, then $x \longrightarrow a b \longrightarrow y$ is a path of length 2. In particular, if we let $x=y$, then $g(\Gamma(R))$ is equal to or less than 3 .

Example 1 (See Example 1.5, p. 5 in [5]). Let

$$
R=\left\{\left(\begin{array}{ll}
\mathbb{Z} & \mathbb{Z} / 2 \mathbb{Z} \\
0 & \mathbb{Z} / 2 \mathbb{Z}
\end{array}\right)\right\} \text { and take } a=\left(\begin{array}{ll}
2 & 0 \\
0 & \overline{1}
\end{array}\right) \in R
$$


Since the left annihilator of $a$ is equal to $\{0\}$ but the right annihilator of $a$ is not equal to $\{0\}, a$ is not a left zero-divisor, and so $a$ is an origin but $a$ is a right zero-divisor. Since there is no path from $a$ to $a^{2}, \Gamma(R)$ is not connected.

Let

$$
S=\left\{\left(\begin{array}{cc}
\mathbb{Z} & 0 \\
\mathbb{Z} / 2 \mathbb{Z} & \mathbb{Z} / 2 \mathbb{Z}
\end{array}\right)\right\} \text { and take } c=\left(\begin{array}{ll}
2 & 0 \\
0 & \overline{1}
\end{array}\right) \in S .
$$

Similarly, we note that $c$ is not a right zero-divisor, and so $c$ is a $\operatorname{sink}$ but $c$ is a left zero-divisor. Since there is also no path from $c^{2}$ to $c, \Gamma(S)$ is not connected.

Remark 2. In [3, Theorem 2.3], Anderson and Livingston have shown that for every commutative ring $R, \Gamma(R)$ is connected and $\operatorname{diam}(\Gamma(R))$ is equal to or less than 3. But by Example 1 we can note that there is a noncommutative ring in which its zero-divisor graph is not connected and also note that the condition [there are no sources and no sinks in the zero-divisor graph of a noncommutative ring] is not superfluous to be connected.

Theorem 2.3. Let $R$ be a ring such that $X$ is a union of finite number of orbits under the left and right regular action on $X$ by $G$. Then $X=Z^{*}(R)$, and so $\Gamma_{X}(R)$ is connected and $\operatorname{diam}\left(\Gamma_{X}(R)\right)$ (resp. $\left.g(\Gamma(R))\right)$ is equal to or less than 3.

Proof. Since $X$ is a union of finite number of orbits under the left regular action on $X$ by $G$, then $Z_{\ell}^{*}(R) \subseteq Z_{r}^{*}(R)=X$ by [8, Theorem 2.2]. Similarly, we can show that if $X$ is a union of finite number of orbits under the right regular action on $X$ by $G$, then $Z_{r}^{*}(R) \subseteq Z_{\ell}^{*}(R)=X$. Thus $Z^{*}(R)=Z_{\ell}^{*}(R)=Z_{r}^{*}(R)=X$, which implies that $\Gamma(R)$ has no sources and no sinks, and so $\Gamma_{X}(R)$ is connected and $\operatorname{diam}\left(\Gamma_{X}(R)\right)$ (resp. $\left.g(\Gamma(R))\right)$ is equal to or less than 3 by Theorem 2.2.

Corollary 2.4. Let $R$ be a ring such that $X \neq \emptyset$. If $X$ is finite, then $X=$ $Z^{*}(R)$, and so $R$ is finite and $(|X|+1)^{2} \geq|R|$.

Proof. Since $X \neq \emptyset$ and is finite, $X$ is a union of finite number of orbits under the left and right regular action on $X$ by $G$, and so we have $X=Z^{*}(R)$ by the argument given in the proof of Theorem 2.3. Hence $R$ is finite and then $(|X|+1)^{2} \geq|R|$ by $[11$, Theorem I].

Corollary 2.5. Let $R$ be a finite ring. Then $\Gamma_{X}(R)$ is connected and

$$
\operatorname{diam}\left(\Gamma_{X}(R)\right)
$$

(resp. $g(\Gamma(R)))$ is equal to or less than 3.

Proof. Since $R$ is a finite ring, $X$ is a union of finite number of orbits under the left and right regular action on $X$ by $G$. Hence it follows from Theorem 2.3.

Proposition 2.6. Let $n$ be any positive integer and $R$ be the matrix ring of all $n \times n$ matrices over a division ring $D$. Then $X=Z^{*}(R)$, and so $\Gamma_{X}(R)$ is connected and $\operatorname{diam}\left(\Gamma_{X}(R)\right)$ (resp. $\left.g(\Gamma(R))\right)$ is equal to or less than 3. 
Proof. Let $x \in X$ be arbitrary. Then there exists $y \in X$ (resp. $z \in X$ ) such that $x y=0($ resp. $z x=0)$, which implies that $\operatorname{ann}_{r}^{*}(x) \neq \emptyset\left(\operatorname{resp} \operatorname{ann}_{\ell}^{*}(x) \neq \emptyset\right)$ for all $x \in X$, i.e., $X=Z^{*}(R)$. Hence $\Gamma_{X}(R)$ is connected and $\operatorname{diam}(\Gamma(R))$ (resp. $g(\Gamma(R))$ ) is equal to or less than 3 by Theorem 2.2.

Lemma 2.7. Let $R$ and $S$ be two rings. If $\Gamma(R)$ and $\Gamma(S)$ have no sources (resp. no sinks), then $\Gamma(R \times S)$ has no sources (resp. no sinks).

Proof. Let $\left(x_{R}, x_{S}\right) \in Z^{*}(R \times S)$ be arbitrary. Then $x_{R} \in Z^{*}(R)$ or $x_{S} \in$ $Z^{*}(S)$. If $x_{R} \in Z^{*}(R)$, then there is $y_{R} \in X(R)$ such that $y_{R} x_{R}=0_{R}$ where $0_{R}$ is the additivite identity of $R$ since $\Gamma(R)$ has no origins. Thus $\left(y_{R}, 0_{S}\right)\left(x_{R}, x_{S}\right)=\left(0_{R}, 0_{S}\right)$ where $0_{S}$ is the additivite identity of $S$, and so $\Gamma(R \times S)$ has no sources. Similarly, if $x_{S} \in Z^{*}(S)$, then $\Gamma(R \times S)$ has no sources. By the similar argument, if $\Gamma(R)$ and $\Gamma(S)$ have no sinks, then $\Gamma(R \times S)$ has no sinks.

Corollary 2.8. Let $R_{1}, R_{2}, \ldots, R_{n}$ be rings for some positive integer $n$. If all $\Gamma\left(R_{i}\right)$ for $i=1,2, \ldots, n$ have no sources (resp. sinks), then $\Gamma\left(R_{1} \times R_{2} \times \cdots \times R_{n}\right)$ has no sources (resp. no sinks).

Proof. It follows from the Lemma 2.7 and the mathematical induction on $n$.

Proposition 2.9. Let $R$ be a ring with $X=o_{r}(x) \cup o_{r}\left(x^{2}\right) \cup \cdots \cup o_{r}\left(x^{n}\right)$ (resp. $\left.X=o_{\ell}(x) \cup o_{\ell}\left(x^{2}\right) \cup \cdots \cup o_{\ell}\left(x^{n}\right)\right)$ under the right (resp. left) regular action on $X$ by $G$ for some positive integer $n$. If $n=1$ and $|X| \geq 3$, or $n=2$ and $o_{r}\left(x^{2}\right) \neq\left\{x^{2}\right\}$, or $n=3$ and $o_{r}\left(x^{i}\right) \neq\left\{x^{i}\right\}$ for some $i=2$ or 3 , or $n \geq 4$, then there exists a cycle of length 3 in $\Gamma(R)$.

Proof. Consider the right regular action of $G$ on $X$. If $n=1$, right regular action is transitive, then $\Gamma(R)$ is complete by Proposition 2.1. Since $|X| \geq 3$, there exists a cycle of length 3 in $\Gamma(R)$. If $n=2$ and $o_{r}\left(x^{2}\right) \neq\left\{x^{2}\right\}$, then there exists $g \in G$ such that $x^{2} g \neq x^{2}$. Since $X=o(x) \cup o\left(x^{2}\right)$ and $x^{2} g \in X$, $x^{2} g=h x$ or $h x^{2}$ for some $h \in G$. Thus $x^{2} \longrightarrow x \longrightarrow x^{2} g \longrightarrow x^{2}$ is a cycle of length 3. If $n=3$ and $o_{r}\left(x^{i}\right) \neq\left\{x^{i}\right\}$ for some $i=2$ or 3 , then there exists $g \in G$ such that $x^{i} g \neq x^{i}$. Since $X=o(x) \cup o\left(x^{2}\right) \cup o\left(x^{3}\right)$ and $x^{i} g \in X, x^{i} g=h x$ or $h x^{2}$ or $h x^{3}$ for some $h \in G$. Thus $x^{3} \longrightarrow x^{2} \longrightarrow x^{i} g \longrightarrow x^{3}$ is a cycle of length 3. Finally, if $n \geq 4$, then clearly $x^{n-2} \longrightarrow x^{n-1} \longrightarrow x^{n} \longrightarrow x^{n-2}$ is a cycle of length 3. Similarly, the result holds under the left regular action of $G$ on $X$.

Remark 3. Let $R$ be a ring. Then for each $x \in X, \operatorname{ann}_{\ell}^{*}(x)\left(\operatorname{resp} \operatorname{ann}_{r}^{*}(x)\right)$ is a union of orbits under the left (resp. right) regular action on $X$ by $G$. Indeed, let $y \in \operatorname{ann}_{\ell}^{*}(x)$ be arbitrary. Then we have $o_{\ell}(y) \subseteq \operatorname{ann}_{\ell}^{*}(x)$, and so $\bigcup_{y \in \operatorname{ann}_{\ell}^{*}(x)} o_{\ell}(y) \subseteq \operatorname{ann}_{\ell}^{*}(x)$. Clearly, $\operatorname{ann}_{\ell}^{*}(x) \subseteq \bigcup_{y \in \operatorname{ann}_{\ell}^{*}(x)} o_{\ell}(y)$. Hence $\operatorname{ann}_{\ell}^{*}(x)=\bigcup_{y \in \operatorname{ann}_{\ell}^{*}} o_{\ell}^{*}(y)$, i.e., $\operatorname{ann}_{\ell}^{*}(x)$ is a union of orbits under the left regular action on $X$ by $G$. By the similar argument, $\operatorname{ann}_{r}^{*}(x)$ is a union of orbits under the right regular action on $X$ by $G$. 
Theorem 2.10. Let $R$ be a ring such that $X$ is a union of finite number of orbits under the left (resp. right) regular action on $X$ by $G$. If $R$ is a local ring, then there is a vertex of $\Gamma_{X}(R)$ which is adjacent to every other vertex in $\Gamma_{X}(R)$.

Proof. Let $X$ be a union of $n$ orbits under the left (resp. right) regular action on $X$ by $G$. Since $R$ is a local ring, by [8, Lemma 2.3] there exists $x \in X$ such that $x^{n} \neq 0=x^{n+1}$ and $X=o_{\ell}(x) \cup o_{\ell}\left(x^{2}\right) \cup \cdots \cup o_{\ell}\left(x^{n}\right)$. Hence we have $\operatorname{ann}_{\ell}\left(x^{n}\right)=X$, i.e., $a \longrightarrow x^{n}$ for all $a \in X$, which means that $x^{n}$ is adjacent to every other vertex in $\Gamma_{X}(R)$. By the similar argument, we can show that if $X$ is a union of $n$ orbits under the right regular action on $X$ by $G$, then there exists $y \in X$ such that $y^{n} \neq 0=y^{n+1}$ and $X=o_{r}(y) \cup o_{r}\left(y^{2}\right) \cup \cdots \cup o_{r}\left(y^{n}\right)$. Thus $\operatorname{ann}_{r}\left(y^{n}\right)=X$, i.e., $y^{n} \longrightarrow b$ for all $b \in X$, which means that $y^{n}$ is adjacent to every other vertex in $\Gamma_{X}(R)$.

Remark 4. We note that in the proof of Theorem 2.11 if $R$ is a local ring such that $X=o_{\ell}(x) \cup o_{\ell}\left(x^{2}\right) \cup \cdots \cup o_{\ell}\left(x^{n}\right)\left(\right.$ resp. $\left.X=o_{r}(x) \cup o_{r}\left(x^{2}\right) \cup \cdots \cup o_{r}\left(x^{n}\right)\right)$ with $x^{n} \neq 0=x^{n+1}$ under the left (resp. right) regular action on $X$ by $G$, then the subgraph $\Gamma_{o_{\ell}\left(x^{n}\right)}\left(\operatorname{resp} . \Gamma_{o_{r}\left(x^{n}\right)}\right)$ of $\Gamma_{X}(R)$ is complete.

Corollary 2.11. If $R$ is a finite local ring, then there is a vertex of $\Gamma_{X}(R)$ which is adjacent to every other vertex in $\Gamma_{X}(R)$.

Proof. Since $R$ is a finite ring, $X$ is a union of finite number of orbits under the left and right regular action on $X$ by $G$. Hence it follows from Theorem 2.10.

Recall that a ring $R$ is called unit-regular if for every $x \in R$ there exists a unit $g \in R$ such that $x g x=x$. In [10], it has been shown that $R$ is a unit-regular ring if and only if for every orbit $o_{\ell}(x)(x \in X)$ under the left regular action on $X$ by $G$, there exists some idempotent $e \in X$ such that $o_{\ell}(x)=o_{\ell}(e)$. Similarly, we can show that $R$ is a unit-regular ring if and only if for every orbit $o_{r}(x)(x \in X)$ under the right regular action of $G$ on $X$, there exists some idempotent $e \in X$ such that $o_{r}(x)=o_{r}(e)$.

Proposition 2.12. Let $R$ be a unit-regular ring such that $X \neq \emptyset$. Then $\Gamma_{X}(R)$ is connected and $\operatorname{diam}\left(\Gamma_{X}(R)\right)$ (resp. $\left.g(\Gamma(R))\right)$ is equal to or less than 3.

Proof. Let $x \in X$ be arbitrary. Then there exists an idempotent $e_{1} \in X$ such that $o_{\ell}(x)=o_{\ell}\left(e_{1}\right)$ under the left regular action on $X$ by $G$ by $[10$, Lemma 2.3]. By the similar argument, there exists an idempotent $e_{2} \in X$ such that $o_{r}(x)=o_{r}\left(e_{2}\right)$ under the right regular action on $X$ by $G$. Hence there exists $g_{1} \in G$ (resp. $g_{2} \in G$ ) such that $x=g_{1} e_{1}$ (resp. $x=e_{2} g_{2}$ ). Since $x\left(1-e_{1}\right)=g_{1} e_{1}\left(1-e_{1}\right)=0$ (resp. $\left(1-e_{2}\right) x=\left(1-e_{2}\right) e_{2} g_{2}=0, x$ is neither source nor sink. Thus $\Gamma_{X}(R)$ is connected and $\operatorname{diam}\left(\Gamma_{X}(R)\right)$ is equal to or less than 3 by Theorem 2.2 . 
Proposition 2.13. Let $R$ be a unit-regular ring. Then $\Gamma_{X}(R)$ is complete if and only if the set of all idempotents in $R$ is orthogonal and the left regular action on $X$ by $G$ is trivial, i.e., $o_{\ell}(x)=\{x\}$ for all $x \in X$.

Proof. $(\Rightarrow)$ Suppose that $\Gamma_{X}(R)$ is complete. Clearly, the set of all idempotents in $R$ is orthogonal. Assume that the left regular action of $G$ on $X$ is not trivial. Then there exists an idempotent $e \in X$ such that $o_{\ell}(e) \neq\{e\}$ by $[10$, Lemma 2.3] and so there exists $y(\neq e) \in o_{\ell}(e)$ such that $y=g e$ for some $g \in G$. Since $\Gamma_{X}(R)$ is complete and $y, e(y \neq e) \in X, 0=y e=(g e) e=g e=y$, a contradiction. Hence the left regular action on $X$ by $G$ is trivial.

$(\Leftarrow)$ It follows from [10, Lemma 2.3].

Corollary 2.14. Let $R$ be a unit-regular ring. Then $\Gamma_{X}(R)$ is complete if and only if the set of all idempotents in $R$ is orthogonal and the right regular action on $X$ by $G$ is trivial, i.e., $o_{r}(x)=\{x\}$ for all $x \in X$.

Proof. It follows from the similar argument given in the proof of Proposition 2.13 .

Lemma 2.15. Let $R$ be a ring. If under the left (resp. right) regular action on $X$ by $G, y \in o_{\ell}(x)$ (resp. $y \in o_{r}(x)$ ) for some $x \in X$, then $\operatorname{ann}_{r}(x)=\operatorname{ann}_{r}(y)$ (resp. $\left.\operatorname{ann}_{\ell}(x)=\operatorname{ann}_{\ell}(y)\right)$.

Proof. If $y \in o_{\ell}(x)$ (resp. $\left.y \in o_{r}(x)\right)$ for some $x \in X$, then there exists $g \in G$ (resp. $h \in G$ ) such that $y=g x$ (resp. $y=x h$ ). It is obvious to show that $\operatorname{ann}_{r}(x)=\operatorname{ann}_{r}(y)\left(\operatorname{resp} . \operatorname{ann}_{\ell}(x)=\operatorname{ann}_{\ell}(y)\right)$.

Corollary 2.16. Let $R$ be a unit-regular ring with $X \neq \emptyset$. Then for any $x \in X$ there exists an idempotent $e \in X$ such that $\operatorname{ann}_{r}(x)=\operatorname{ann}_{r}(e)$ (resp. $\left.\operatorname{ann}_{\ell}(x)=\operatorname{ann}_{\ell}(e)\right)$.

Proof. It follows from the Lemma 2.15 and [10, Lemma 2.3].

Proposition 2.17. Let $R$ be a unit-regular ring such that $X \neq \emptyset$ and $2=2 \cdot 1$ is a unit in $R$. Then there exists a cycle of length 4 in $\Gamma(R)$.

Proof. Let $e \in X$ be an idempotent. Since $2=2 \cdot 1 \in G, e \neq 1-e,-e$. Thus $e \longrightarrow 1-e \longrightarrow-e \longrightarrow e-1 \longrightarrow e$ is a cycle of length 4 in $\Gamma(R)$.

\section{Automorphism of graph over $\operatorname{Mat}_{2}\left(\mathbb{Z}_{p}\right)$}

Recall that a graph automorphism $f$ of a graph $\Gamma(R)$ is a bijection $f$ : $\Gamma(R) \longrightarrow \Gamma_{X}(R)$ which preserves adjacency. Of course, the set $\operatorname{Aut}(\Gamma(R))$ of all graph automorphisms of $\Gamma(R)$ forms a group under the usual composition of functions. In [3], Anderson and Livingston computed $\operatorname{Aut}\left(\Gamma\left(\mathbb{Z}_{n}\right)\right)$. In this section, we compute $\operatorname{Aut}\left(\Gamma\left(\operatorname{Mat}_{2}\left(\mathbb{Z}_{p}\right)\right)\right.$ where $\operatorname{Mat}_{2}\left(\mathbb{Z}_{p}\right)$ is the matrix ring of all $2 \times 2$ matrices over $\mathbb{Z}_{p}$ for any prime $p$. 
Lemma 3.1. Let $R$ be a ring and $f: \Gamma_{X}(R) \longrightarrow \Gamma_{X}(R)$ be a graph automorphism of $\Gamma_{X}(R)$. Then for all $x \in X, f\left(\operatorname{ann}_{\ell}(x)\right)=\operatorname{ann}_{\ell}(f(x))$ (resp. $\left.f\left(\operatorname{ann}_{r}(x)\right)=\operatorname{ann}_{r}(f(x))\right)$.

Proof. Let $y \in f\left(\operatorname{ann}_{\ell}(x)\right)$ be arbitrary. Then $y=f(z)$ for some $z \in \operatorname{ann}_{\ell}(x)$. Since $z x=0,0=f(z x)=f(z) f(x)=y f(x)$ and so $y \in \operatorname{ann}_{\ell}(f(x))$. Hence $f\left(\operatorname{ann}_{\ell}(x)\right) \subseteq \operatorname{ann}_{\ell}(f(x))$. Let $z \in \operatorname{ann}_{\ell}(f(x))$ be arbitrary. Then $z f(x)=$ 0 . Since $f$ is one to one, there exists $z_{1} \in X$ such that $f\left(z_{1}\right)=z$. Then $0=z f(x)=f\left(z_{1}\right) f(x)=f\left(z_{1} x\right)$, and so $z_{1} x=0$. Since $z_{1} \in \operatorname{ann}_{\ell}(x)$ and $z=f\left(z_{1}\right) \in f\left(\operatorname{ann}_{\ell}(x)\right), \operatorname{ann}_{\ell}(f(x)) \subseteq f\left(\operatorname{ann}_{\ell}(x)\right)$. By the similar argument, we have $f\left(\operatorname{ann}_{r}(x)\right)=\operatorname{ann}_{r}(f(x))$.

In a ring $R$ with identity the left (resp. right) regular action of $G$ on $X$ is said to be half-transitive if $G$ is transitive on $X$ or if $o_{\ell}(x)\left(\operatorname{resp} . o_{r}(x)\right)$ is a finite set with $\left|o_{\ell}(x)\right|>1$ (resp. $\left|o_{r}(x)\right|>1$ ) and $\left|o_{\ell}(x)\right|=\left|o_{\ell}(y)\right|$ (resp. $\left.\left|o_{r}(x)\right|=\left|o_{r}(y)\right|\right)$ for all $x$ and $y \in X$. In [9, Theorem 2.4 and Lemma 2.7], it was shown that if $R$ is a matrix ring of all $2 \times 2$ matrices over a finite field $F$, then $G$ is half-transitive on $X$ by the left (resp. right) regular action and $\left|o_{\ell}(x)\right|=|F|^{2}-1\left(\right.$ resp. $\left.\left|o_{r}(x)\right|=|F|^{2}-1\right)$ for all $x \in X$.

Lemma 3.2. Let $p$ be a prime and $R=\operatorname{Mat}_{2}\left(\mathbb{Z}_{p}\right)$. Then for any $x \in X$, $\operatorname{ann}_{\ell}^{*}(x)=o_{r}(y)\left(\right.$ resp. $\left.\left.\operatorname{ann}_{r}^{*}(x)\right)=o_{\ell}(z)\right)$ for some $y \in X($ resp. $z \in X)$.

Proof. By [9, Lemma 2.7], we have $\left|o_{\ell}(x)\right|=p^{2}-1$ (resp. $\left|o_{r}(x)\right|=p^{2}-1$ ) for all $x \in X$. Since $\operatorname{ann}_{\ell}^{*}(x)\left(\operatorname{resp} . \operatorname{ann}_{r}^{*}(x)\right)$ is a union of a finite number of orbits under the left (resp. right) regular action of $G$ on $X$ by Remark 3 and since the left (resp. right) regular action of $G$ on $X$ is half-transitive by [9, Theorem 2.4], $\left|o_{\ell}(y)\right|\left(\operatorname{resp} .\left|o_{r}(z)\right|\right)$ for all $y \in \operatorname{ann}_{\ell}^{*}(x)\left(\operatorname{resp}\right.$. all $\left.z \in \operatorname{ann}_{r}^{*}(x)\right)$ is a divisor of $\left|\operatorname{ann}_{\ell}^{*}(x)\right|\left(\operatorname{resp} .\left|\operatorname{ann}_{r}^{*}(x)\right|\right)$ and then $\left|\operatorname{ann}_{\ell}^{*}(x)\right|=p^{2}-1$ or $p^{3}-1$ (resp. $\left|\operatorname{ann}_{r}^{*}(x)\right|=p^{2}-1$ or $\left.p^{3}-1\right)$ since $\left|\operatorname{ann}_{l}(x)\right|=p^{2}$ or $p^{3}\left(\operatorname{resp} .\left|\operatorname{ann}_{r}(x)\right|=p^{2}\right.$ or $\left.p^{3}\right)$ and so $\left|\operatorname{ann}_{\ell}^{*}(x)\right|=p^{2}-1\left(\operatorname{resp} . \mid \operatorname{ann}_{r}^{*}(x)=p^{2}-1\right)$. Hence we have the result.

Lemma 3.3. Let $p$ be a prime and $R=\operatorname{Mat}_{2}\left(\mathbb{Z}_{p}\right)$. Then the number of orbits under the left (resp. right) regular action on $X$ by $G$ is $p+1$.

Proof. Let $\mu$ be the number of orbits under the left (resp. right) regular action on $X$ by $G$. Note that $|G|=\left(p^{2}-1\right)\left(p^{2}-p\right)$. Thus $|X|=|R|-|G|-1=$ $p^{4}-\left(p^{2}-1\right)\left(p^{2}-p\right)-1=(p+1)\left(p^{2}-1\right)$. Since the cardinality of any orbit under the left (resp. right) regular action on $X$ by $G$ is $p^{2}-1$ by $[9$, Lemma 2.7], $\mu=|X| /\left(p^{2}-1\right)=p+1$.

Lemma 3.4. Let $p$ be a prime, $R=\operatorname{Mat}_{2}\left(\mathbb{Z}_{p}\right)$ and let $N(p)$ be the set of nonzero nilpotents in $R$. Then $|N(p)|=p^{2}-1$.

Proof. Let

$$
N_{1}(p)=\left\{\left(\begin{array}{cc}
a & b \\
\alpha a & \alpha b
\end{array}\right) \in N(p) \mid a, b, \alpha \neq 0\right\}
$$


and

$$
N_{2}(p)=\left\{\left(\begin{array}{cc}
a & -\alpha a \\
b & -\alpha b
\end{array}\right) \in N(p) \mid a, b, \alpha \neq 0\right\} .
$$

We will show that $N_{1}(p)=N_{2}(p)$. Let

$$
\left(\begin{array}{ll}
a & -\alpha a \\
b & -\alpha b
\end{array}\right) \in N_{2}(p)
$$

be arbitrary. Since $A^{2}=0$ and $a, b \neq 0$, we have

$$
A=\left(\begin{array}{cc}
\alpha b & -\alpha^{2} b \\
b & -\alpha b
\end{array}\right) \in N_{2}(p),
$$

and also $\left(1 / \alpha^{2}\right)\left(\begin{array}{cc}\alpha b & -\alpha^{2} b \\ b & -\alpha b\end{array}\right) \in N_{2}(p)$.

Since

$$
\left(1 / \alpha^{2}\right)\left(\begin{array}{cc}
\alpha b & -\alpha^{2} b \\
b & -\alpha b
\end{array}\right)=\left(\begin{array}{cc}
(-1 / \alpha)(-b) & -b \\
\left(-1 / \alpha^{2}\right)(-b) & (-1 / \alpha)(-b)
\end{array}\right) \in N_{1}(p),
$$

we have $N_{2}(p) \subseteq N_{1}(p)$. By the similar argument, we can have $N_{1}(p) \subseteq N_{2}(p)$.

Let $A$ be any nonzero nilpotent in $R$. Then

$$
A=\left(\begin{array}{cc}
a & b \\
\alpha a & \alpha b
\end{array}\right) \text { or }\left(\begin{array}{cc}
a & \alpha a \\
a & \alpha b
\end{array}\right)
$$

for some $\alpha \in \mathbb{Z}_{p}$.

Note that since $A$ is a nonzero nilpotent in $R, b \neq 0$. Consider the following cases:

Case 1. $\alpha=0$;

Since

$$
A^{2}=0, A=\left(\begin{array}{ll}
0 & b \\
0 & 0
\end{array}\right) \text { or }\left(\begin{array}{ll}
0 & 0 \\
b & 0
\end{array}\right)
$$

for all nonzero $b \in \mathbb{Z}_{p}$.

Case 2. $\alpha \neq 0$;

In this case, $a \neq 0$. Hence we have $N_{1}(p)=N_{2}(p)$ by the above argument. Since $A^{2}=0$, we have $A=\left(\begin{array}{cc}-\alpha b & b \\ -\alpha^{2} b & \alpha b\end{array}\right)$.

Consequently, we have

$$
\begin{aligned}
|N(p)|= & \left|N_{1}(p)\right|+\left|\left\{\left(\begin{array}{ll}
0 & b \\
0 & 0
\end{array}\right) \in N(p): b(\neq 0)\right\}\right| \\
& +\left|\left\{\left(\begin{array}{ll}
0 & 0 \\
b & 0
\end{array}\right) \in N(p): b(\neq 0)\right\}\right| \\
= & (p-1)(p-1)+2(p-1)=p^{2}-1 .
\end{aligned}
$$


Example 2. Let $R=\operatorname{Mat}_{2}\left(\mathbb{Z}_{2}\right)$. Then $X=\left\{x_{1}, x_{2}, x_{3}, x_{4}, x_{5}, x_{6}, x_{7}, x_{8}, x_{9}\right\}$, where

$$
\begin{gathered}
x_{1}=\left(\begin{array}{ll}
0 & 0 \\
0 & 1
\end{array}\right), x_{2}=\left(\begin{array}{ll}
0 & 0 \\
1 & 0
\end{array}\right), x_{3}=\left(\begin{array}{ll}
0 & 0 \\
1 & 1
\end{array}\right), x_{4}=\left(\begin{array}{ll}
0 & 1 \\
0 & 0
\end{array}\right), \\
x_{5}=\left(\begin{array}{ll}
0 & 1 \\
0 & 1
\end{array}\right), x_{6}=\left(\begin{array}{ll}
1 & 0 \\
0 & 0
\end{array}\right), x_{7}=\left(\begin{array}{ll}
1 & 0 \\
1 & 0
\end{array}\right), x_{8}=\left(\begin{array}{ll}
1 & 1 \\
0 & 0
\end{array}\right), x_{9}=\left(\begin{array}{ll}
1 & 1 \\
1 & 1
\end{array}\right) .
\end{gathered}
$$

Note that $\left\{x_{2}, x_{4}, x_{9}\right\}$ is the set of nonzero nilpotents in $R$. Under the left (resp. right) regular action on $X$ by $G$, there are three orbits $o_{\ell}\left(x_{2}\right)=$ $\left\{x_{2}, x_{6}, x_{7}\right\}, o_{\ell}\left(x_{4}\right)=\left\{x_{1}, x_{4}, x_{5}\right\}, o_{\ell}\left(x_{9}\right)=\left\{x_{3}, x_{8}, x_{9}\right\}$ (resp. $o_{r}\left(x_{2}\right)=$ $\left.\left\{x_{1}, x_{2}, x_{3}\right\}, o_{r}\left(x_{4}\right)=\left\{x_{4}, x_{6}, x_{8}\right\}, o_{r}\left(x_{9}\right)=\left\{x_{5}, x_{7}, x_{9}\right\}\right)$.

We can compute $\operatorname{Aut}(\Gamma(R))=\{1, f, g, g \circ f, f \circ g, g \circ f \circ g\}$, where

$$
\begin{aligned}
f & =\left(\begin{array}{lllllllll}
x_{1} & x_{2} & x_{3} & x_{4} & x_{5} & x_{6} & x_{7} & x_{8} & x_{9} \\
x_{3} & x_{2} & x_{1} & x_{9} & x_{7} & x_{5} & x_{8} & x_{6} & x_{4}
\end{array}\right) \\
g & =\left(\begin{array}{lllllllll}
x_{1} & x_{2} & x_{3} & x_{4} & x_{5} & x_{6} & x_{7} & x_{8} & x_{9} \\
x_{6} & x_{4} & x_{8} & x_{2} & x_{1} & x_{3} & x_{7} & x_{5} & x_{9}
\end{array}\right) \text { are permutations. }
\end{aligned}
$$

Observe that $\operatorname{Aut}(\Gamma(R))$ is isomorphic to $S_{3}$, the symmetric group of degree 3 .

Theorem 3.5. Let $p$ be a prime and $R=\operatorname{Mat}_{2}\left(\mathbb{Z}_{p}\right)$. Then $\operatorname{Aut}(\Gamma(R)) \neq\{1\}$.

Proof. If $p=2$, then $\operatorname{Aut}(\Gamma(R)) \neq\{1\}$ by Example 2. Suppose that $p \geq 3$. Let $N(p)$ be the set of nonzero nilpotents in $R$. Since the number orbits is $p+1$ by Lemma 3.3 under the left (resp. right) regular action on $X$ by $G$ and $|N(p)|=p^{2}-1$ by Lemma 3.4, there exists $x \in X$ such that $\left|o_{\ell}(x) \cap N(p)\right| \geq 2$. Let $x_{1}, x_{2} \in o_{l}(x) \cap N(p)\left(x_{1} \neq x_{2}\right)$. Since $x_{1}$ and $x_{2}$ are nilpotents, we have $\operatorname{ann}_{\ell}^{*}\left(x_{1}\right)=o_{\ell}\left(x_{1}\right)=o_{\ell}\left(x_{2}\right)=\operatorname{ann}_{\ell}^{*}\left(x_{2}\right)$ by Lemma 3.2. We have also $\operatorname{ann}_{r}^{*}\left(x_{1}\right)=\operatorname{ann}_{r}^{*}\left(x_{2}\right)$. Indeed, if $a \in \operatorname{ann}_{r}^{*}\left(x_{1}\right)$, then $0=x_{1} a=g x_{2} a=0$ for some $g \in G$ since $x_{2} \in o_{\ell}\left(x_{1}\right)$, which implies that $a \in \operatorname{ann}_{r}^{*}\left(x_{2}\right)$, and so $\operatorname{ann}_{r}^{*}\left(x_{1}\right) \subseteq \operatorname{ann}_{r}^{*}\left(x_{2}\right)$. By the similar argument, we have $\operatorname{ann}_{r}^{*}\left(x_{2}\right) \subseteq \operatorname{ann}_{r}^{*}\left(x_{1}\right)$. Also we have $\operatorname{ann}_{r}^{*}\left(x_{1}\right)=o_{r}\left(x_{1}\right)=o_{r}\left(x_{2}\right)=\operatorname{ann}_{r}^{*}\left(x_{2}\right)$ by Lemma 3.2. Let $f=\left(x_{1}, x_{2}\right)$ be a transposition in $S_{|X|}$, the symmetric group of degree $|X|$. Since $x_{1} \neq x_{2}, f \neq 1$. We will show that $f \in \operatorname{Aut}(\Gamma(R))$. Consider $x_{1} y=0$ for some $y \in X$. If $y=x_{1}$, then $f\left(x_{1}\right) f(y)=x_{2} x_{2}=0$. If $y=x_{2}$, then $f\left(x_{1}\right) f(y)=x_{2} x_{1}=g_{1} x_{1} x_{1}=0$ for some $g_{1} \in G$ since $x_{2} \in o_{l}\left(x_{1}\right)$. If $y \neq$ $x_{1}, x_{2}$, then $f\left(x_{1}\right) f(y)=x_{2} y=g_{1} x_{1} y=0$ for some $g_{1} \in G$ since $x_{2} \in o_{l}\left(x_{1}\right)$. Also consider $z x_{1}=0$ for some $z \in X$. If $z=x_{1}$, then $f(z) f\left(x_{1}\right)=x_{2} x_{2}=0$. If $z=x_{2}$, then $f(z) f\left(x_{1}\right)=x_{1} x_{2}=h_{1} x_{2} x_{2}=0$ for some $h_{1} \in G$ since $x_{1} \in o\left(x_{2}\right)$. If $z \neq x_{1}, x_{2}$, then $f(z) f\left(x_{1}\right)=z x_{2}=z x_{1} h_{2}=0$ for some $h_{2} \in G$ since $x_{2} \in o_{r}\left(x_{1}\right)$. Consequently, $f \in \operatorname{Aut}(\Gamma(R))$, and so $\operatorname{Aut}(\Gamma(R)) \neq\{1\}$.

Remark 5. Let $p$ be a prime, $R=\operatorname{Mat}_{2}\left(\mathbb{Z}_{p}\right)$ and $N(p)$ be the set of nonzero nilpotents in $R$. We can choose that $f(\neq 1) \in \operatorname{Aut}(\Gamma(R))$ by Theorem 3.5. Then we note that (1) $f(a) \in N(p)$ for all $a \in N(p)$; (2) since $f$ is bijective 
and the left (resp. right) regular action on $X$ by $G$ is half-transitive with $\left|o_{\ell}(x)\right|=p+1$ (resp. $\left|o_{r}(x)\right|=p+1$ ) for all $x \in X,\left|o_{l}(x) \cap N(p)\right|=p-1$ (resp. $\left.\left|o_{r}(x) \cap N(p)\right|=p-1\right)$ and $f\left(o_{\ell}(x)\right)=o_{\ell}(f(x))$ (resp. $\left.f\left(o_{r}(x)\right)=o_{r}(f(x))\right)$ by Lemma 3.1 and Lemma 3.2; (3) every orbit under the left (resp. right) regular action on $X$ by $G$ is $o_{\ell}(x)$ (resp. $\left.o_{r}(x)\right)$ for some nilpotent $x \in X$.

Lemma 3.6. Let $p$ be a prime, $R=\operatorname{Mat}_{2}\left(\mathbb{Z}_{p}\right)$ and $N(p)$ be the set of all nonzero nilpotents in $R$. Then under the left (resp. right) regular action on $X$ by $G, o_{\ell}(a) \cap N(p)=o_{r}(a) \cap N(p)=o_{\ell}(a) \cap o_{r}(a)$ for all $a \in N(p)$.

Proof. Let $b \in o_{\ell}(a) \cap N(p)$ be arbitrary. Since $o_{\ell}(a)=o_{\ell}(b), b a=a b=0$, and thus $b \in \operatorname{ann}_{r}^{*}(a)=o_{r}(a)$. Hence $o_{\ell}(a) \cap N(p) \subseteq o_{r}(a) \cap N(p)$ and $o_{\ell}(a) \cap N(p) \subseteq$ $o_{\ell}(a) \cap o_{r}(a)$. By the similar argument, we have $o_{r}(a) \cap N(p) \subseteq o_{\ell}(a) \cap N(p)$ and $o_{r}(a) \cap N(p) \subseteq o_{\ell}(a) \cap o_{r}(a)$. Therefore, $o_{\ell}(a) \cap N(p)=o_{r}(a) \cap N(p) \subseteq$ $o_{\ell}(a) \cap o_{r}(a)$. By Remark 4, we already knew that $\left|o_{r}(a) \cap N(p)\right|=\mid o_{\ell}(a) \cap$ $N(p) \mid=p-1$. Next, we will show that $o_{\ell}(a) \cap N(p)=o_{\ell}(a) \cap o_{r}(a)$. Let $S=\operatorname{ann}_{\ell}(a) \cap \operatorname{ann}_{r}(a)$. Then $S=\left(o_{\ell}(a) \cap o_{r}(a)\right) \cup\{0\}$. Since $S$ is an additive subgroup of $\operatorname{ann}_{\ell}(a)$ and $\left|\operatorname{ann}_{\ell}(a)\right|=p^{2},|S|=1$ or $p$. Since $\left|o_{\ell}(a) \cap o_{r}(a)\right| \geq$ $\left|o_{\ell}(a) \cap N(p)\right|=p-1 \geq 1,|S|=\left|o_{\ell}(a) \cap o_{r}(a)\right|+1 \geq 2$, and thus $|S|=p$. Since $\left|o_{\ell}(a) \cap o_{r}(a)\right|=|S|-1=p-1=\left|o_{\ell}(a) \cap N(p)\right|=\left|o_{r}(a) \cap N(p)\right|$ and $o_{\ell}(a) \cap N(p), o_{r}(a) \cap N(p) \subseteq o_{\ell}(a) \cap o_{r}(a)$, we have $o_{\ell}(a) \cap N(p)=o_{r}(a) \cap N(p)=$ $o_{\ell}(a) \cap o_{r}(a)$.

Remark 6 . Let $p$ be a prime, $R=\operatorname{Mat}_{2}\left(\mathbb{Z}_{p}\right)$ and $N(p)$ be the set of nonzero nilpotents in $R$. We can choose $a_{1}, \ldots, a_{p+1} \in N(p)$ such that $X=o_{\ell}\left(a_{1}\right) \cup \cdots \cup$ $o_{\ell}\left(a_{p+1}\right)$ (resp. $\left.X=o_{r}\left(a_{1}\right) \cup \cdots \cup o_{r}\left(a_{p+1}\right)\right)$. Note that for each $i=1, \ldots, p+1$, $o_{\ell}\left(a_{i}\right)=o_{\ell}\left(a_{i}\right) \cap X=o_{\ell}\left(a_{i}\right) \cap\left[o_{r}\left(a_{1}\right) \cup \cdots \cup o_{r}\left(a_{p+1}\right)\right]=\left[o_{\ell}\left(a_{i}\right) \cap o_{r}\left(a_{1}\right)\right] \cup \cdots \cup$ $\left[o_{\ell}\left(a_{i}\right) \cap o_{r}\left(a_{p+1}\right)\right]$.

Lemma 3.7. Let $p$ be a prime, $R=\operatorname{Mat}_{2}\left(\mathbb{Z}_{p}\right)$ and $N(p)$ be the set of nonzero nilpotents in $R$. Consider $X=o_{\ell}\left(a_{1}\right) \cup \cdots \cup o_{\ell}\left(a_{p+1}\right)$ (resp. $X=o_{r}\left(a_{1}\right) \cup$ $\left.\cdots \cup o_{r}\left(a_{p+1}\right)\right)$ for some $a_{1}, \ldots, a_{p+1} \in N(p)$ as mentioned in Remark 6 . Then under the left (resp. right) regular action on $X$ by $G,\left|o_{\ell}\left(a_{i}\right) \cap o_{r}\left(a_{j}\right)\right|=p-1$ for all $a_{i}, a_{j} \in N(p)(i, j=1, \ldots, p+1)$.

Proof. Let $A_{i j}=\operatorname{ann}_{\ell}\left(a_{i}\right) \cap \operatorname{ann}_{r}\left(a_{j}\right)$ for all $i, j=1, \ldots, p+1$. Note that $A_{i j}=\left[o_{e l l}\left(a_{i}\right) \cap o_{r}\left(a_{j}\right)\right] \cup\{0\}$. If $i=j$, then $\left|o_{l}\left(a_{i}\right) \cap o_{r}\left(a_{j}\right)\right|=p-1$ as given in the proof of Lemma 3.6. Suppose that $i \neq j$. Since $A_{i j}$ ia an additive subgroup of $\operatorname{ann}_{\ell}\left(a_{i}\right)$ with $\left|\operatorname{ann}_{\ell}\left(a_{i}\right)\right|=p^{2},\left|A_{i j}\right|=1$ or $p$. Hence $\left|o_{\ell}\left(a_{i}\right) \cap o_{r}\left(a_{j}\right)\right|=0$ or $p-1$. Assume that $\left|A_{i j}\right|=1$ (equivalently, $\left|o_{\ell}\left(a_{i}\right) \cap o_{r}\left(a_{j}\right)\right|=0$ ) for some $i, j$. Then $\left|A_{i k}\right|>\left|A_{i i}\right|$ for some $k$. Since $\left|A_{i i}\right|=p$ (equivalently, $\left|o_{\ell}\left(a_{i}\right) \cap o_{r}\left(a_{j}\right)\right|=p-1$ ) as given in the proof of Lemma 3.6, $\left|A_{i k}\right|>p$, a contradiction. Therefore, $\left|A_{i j}\right|=p$, and so $\left|o_{\ell}\left(a_{i}\right) \cap o_{r}\left(a_{j}\right)\right|=p-1$ for all $i, j=1, \ldots, p+1$.

Lemma 3.8. Let $p$ be a prime, $R=\operatorname{Mat}_{2}\left(\mathbb{Z}_{p}\right)$ and $N(p)$ be the set of nonzero nilpotents in $R$. Consider $X=o_{\ell}\left(a_{1}\right) \cup \cdots \cup o_{\ell}\left(a_{p+1}\right)$ (resp. $X=o_{r}\left(a_{1}\right) \cup$ $\left.\cdots \cup o_{r}\left(a_{p+1}\right)\right)$ for some $a_{1}, \ldots, a_{p+1} \in N(p)$ as mentioned in Remark 5. If 
$s_{j}=(1, j)$ is a transposition in $S_{p+1}$, the symmetric group of degree $p+1$, and $f_{s_{j}}: \Gamma(R) \longrightarrow \Gamma(R)$ is a bijective map such that $f_{s_{j}}\left(o_{\ell}\left(a_{i}\right)\right)=o_{\ell}\left(a_{s_{j}(i)}\right)$, then $f_{s_{j}}$ is a graph automorphism in $\Gamma(R)$.

Proof. Note that since $f_{s_{j}}: \Gamma(R) \longrightarrow \Gamma(R)$ is a bijective map such that $f_{s_{j}}\left(o_{\ell}\left(a_{i}\right)\right)=o_{\ell}\left(a_{s_{j}(i)}\right), f_{s_{j}}\left(o_{\ell}\left(a_{i}\right) \cap o_{r}\left(a_{k}\right)\right)=o_{\ell}\left(a_{s_{j}(i)}\right) \cap o_{r}\left(a_{s_{j}(k)}\right)$ for all $i, k=1, \ldots, p+1$.

Let $x, y \in X$ be arbitrary. Consider the following cases.

Case 1. $x, y \in o_{\ell}\left(a_{1}\right) \cap o_{r}\left(a_{1}\right)$.

Since $a_{1}^{2}=0, x y=y x=0$. Note that $f_{s_{j}}(x), f_{s_{j}}(y) \in o_{\ell}\left(a_{j}\right)=o_{r}\left(a_{j}\right)$, and so $f_{s_{j}}(x) f_{s_{j}}(y)=f_{s_{j}}(x y)=f_{s_{j}}(0)=0$ and also $f_{s_{j}}(y) f_{s_{j}}(x)=0$.

Case 2. $x, y \in o_{\ell}\left(a_{j}\right) \cap o_{r}\left(a_{j}\right)$.

By the similar argument given to the case $1, x y=y x=0$ and also $f_{s_{j}}(x) f_{s_{j}}(y)$ $=f_{s_{j}}(y) f_{s_{j}}(x)=0$.

Case 3. $x \in o_{\ell}\left(a_{1}\right) \cap o_{r}\left(a_{1}\right), y \in o_{\ell}\left(a_{1}\right) \cap o_{r}\left(a_{j}\right)(j \neq 1)$.

Then $y x=0$. Note that $f_{s_{j}}(x) \in o_{\ell}\left(a_{j}\right) \cap o_{r}\left(a_{j}\right), f_{s_{j}}(y) \in o_{\ell}\left(a_{j}\right) \cap o_{r}\left(a_{1}\right)$, and so $f_{s_{j}}(y) f_{s_{j}}(x)=0$. Assume that $x y=0$. Then $a_{1} a_{j}=0$, which implies that $o_{\ell}\left(a_{1}\right)=o_{\ell}\left(a_{j}\right)$, a contradiction. Hence $x y \neq 0$. Assume that $f_{s_{j}}(x) f_{s_{j}}(y)=0$. Since $f_{s_{j}}(x) \in o_{\ell}\left(a_{j}\right) \cap o_{r}\left(a_{j}\right), f_{s_{j}}(y) \in o_{\ell}\left(a_{j}\right) \cap o_{r}\left(a_{1}\right), a_{j} a_{1}=0$, which implies that $o_{\ell}\left(a_{1}\right)=o_{\ell}\left(a_{j}\right)$, also a contradiction. Hence we have $f_{s_{j}}(x) f_{s_{j}}(y) \neq 0$.

Case 4. $x \in o_{\ell}\left(a_{j}\right) \cap o_{r}\left(a_{j}\right), y \in o_{\ell}\left(a_{1}\right) \cap o_{r}\left(a_{1}\right)$.

By the similar argument given to the case $3, x y=0$ and also $f_{s_{j}}(x) f_{s_{j}}(y)=0$; $y x \neq 0$ and $f_{s_{j}}(y) f_{s_{j}}(x) \neq 0$.

Case 5. $x \in o_{\ell}\left(a_{1}\right) \cap o_{r}\left(a_{i}\right), y \in o_{\ell}\left(a_{1}\right) \cap o_{r}\left(a_{k}\right),(i, k \neq 1, j)$.

Then $x=g_{1} a_{1}=a_{i} h_{1}, y=g_{2} a_{1}=a_{k} h_{2}$ for some $g_{1}, g_{2}, h_{1}, h_{2} \in G$. If $x y=0$, then $a_{1} a_{k}=0$, which implies that $o_{\ell}\left(a_{1}\right)=o_{\ell}\left(a_{k}\right)$, a contradiction. Hence we have $x y \neq 0$. Since $f(x) \in o_{\ell}\left(a_{j}\right) \cap o_{r}\left(a_{i}\right), f(y) \in o_{\ell}\left(a_{j}\right) \cap o_{r}\left(a_{k}\right)$, we also have $f(x) f(y) \neq 0$. Similarly, we have $y x \neq 0$ and $f(y) f(x) \neq 0$.

Case 6. $x \in o_{\ell}\left(a_{i}\right) \cap o_{r}\left(a_{r}\right), y \in o_{\ell}\left(a_{k}\right) \cap o_{r}\left(a_{t}\right),(i, k, r, s \neq 1, j)$. If $x y=0$, then $a_{i} a_{t}=0$. Since $f(x) \in o_{\ell}\left(a_{i}\right) \cap o_{r}\left(a_{r}\right), f(y) \in o_{\ell}\left(a_{k}\right) \cap o_{r}\left(a_{s}\right)$, $f(x) f(y)=0$. Similarly we have that if $y x=0, f(y) f(x)=0$.

Consequently, $f_{s_{j}}$ is a graph automorphism in $\Gamma(R)$.

Theorem 3.9. Let $p$ be a prime and let $R=\operatorname{Mat}_{2}\left(\mathbb{Z}_{p}\right)$. Then $\operatorname{Aut}(\Gamma(R)) \simeq$ $S_{p+1}$ where $S_{p+1}$ is the symmetric group of degree $p+1$.

Proof. Let $N(p)$ be the set of nonzero nilpotents in $R$. We can choose $a_{1}$, $\ldots, a_{p+1} \in N(p)$ such that $X=o_{\ell}\left(a_{1}\right) \cup \cdots \cup o_{\ell}\left(a_{p+1}\right)$. Define $\sigma: S_{p+1} \longrightarrow$ $\operatorname{Aut}(\Gamma(R))$ by $\sigma(s)=f_{s}$ for all $s \in S_{p+1}$ where $f_{s}\left(o_{\ell}\left(a_{i}\right)\right)=o_{\ell}\left(a_{s(i)}\right)$ for all $i=1, \ldots, p+1$. Then $\sigma$ is well-defined and onto. Indeed, by Lemma 3.1 and Lemma 3.2, we have that if $f \in \operatorname{Aut}(\Gamma(R))$ is arbitrary, then for all $i=1, \ldots, p+1, f\left(o_{\ell}\left(a_{i}\right)\right)=o_{\ell}\left(a_{s(i)}\right)$ for some $s \in S_{p+1}$. Since $S_{p+1}$ is generated by the $p$ transpositions $s_{1}=(1,2), \ldots, s_{p}=(1, p+1)$, and $f_{s_{1}}, \ldots, f_{s_{p}} \in$ 
$\operatorname{Aut}(\Gamma(R))$ by Lemma 3.8, $\operatorname{Aut}(\Gamma(R))$ is generated by the $p$ graph automorphisms $f_{s_{1}}, \ldots, f_{s_{p}} \in \operatorname{Aut}(\Gamma(R))$ where $f_{s_{j}}\left(o_{\ell}\left(a_{i}\right)\right)=o_{\ell}\left(a_{s_{j}(i)}\right)$ for all $i=$ $1, \ldots, p+1$ and $j=1, \ldots, p$. Thus $\left|S_{p+1}\right|=|\operatorname{Aut}(\Gamma(R))|$, which implies that $\sigma$ is a bijective map. Also $\sigma$ is a group homomorphism by observing that for all $s_{i}, s_{j} \in S_{p+1}(i, j=1, \ldots, p)$ and all $o_{\ell}\left(a_{k}\right)(k=1, \ldots, p+1)$, $\left(f_{s_{i}} \circ f_{s_{j}}\right)\left(o_{\ell}\left(a_{k}\right)\right)=f_{s_{i} s_{j}}\left(o_{\ell}\left(a_{k}\right)\right)$. Therefore, $\operatorname{Aut}(\Gamma(R)) \simeq S_{p+1}$.

Acknowledgements. The author thanks the referee for his/her helpful comments for the improvement of the paper, also the author thanks Prof. J. Park at Pusan National University for reading this paper and for kind comments.

\section{References}

[1] S. Akbari and A. Mohammadian, On the zero-divisor graph of a commutative ring, J. Algebra 274 (2004), no. 2, 847-855.

[2] D. F. Anderson, A. Frazier, A. Lauve, and P. S. Livingston, The zero-divisor graph of a commutative ring. II, Ideal theoretic methods in commutative algebra (Columbia, MO, 1999), 61-72, Lecture Notes in Pure and Appl. Math., 220, Dekker, New York, 2001.

[3] D. F. Anderson and P. S. Livingston, The zero-divisor graph of a commutative ring, J. Algebra 217 (1999), no. 2, 434-447.

[4] I. Beck, Coloring of commutative rings, J. Algebra 116 (1988), no. 1, 208-226.

[5] A. W. Chatters and C. R. Hajarnavis, Rings with chain conditions, Research Notes in Mathematics, 44. Pitman (Advanced Publishing Program), Boston, Mass.-London, 1980.

[6] F. DeMeyer and L. DeMeyer, Zero divisor graphs of semigroups, J. Algebra 283 (2005), no. $1,190-198$.

[7] R. Diestel, Graph Theory, Graduate Texts in Mathematics, Vol. 173, Springer-Verlag, New York, 1997.

[8] J. Han, Regular action in a ring with a finite number of orbits, Comm. Algebra 25 (1997), no. 7, 2227-2236.

[9] , Half-transitive group actions in a left Artinian ring, Kyungpook Math. J. 37 (1997), no. 2, 297-303.

[10] $\_$, Group actions in a unit-regular ring, Comm. Algebra 27 (1999), no. 7, 33533361.

[11] N. Ganesan, Properties of rings with a finite number of zero divisors. II, Math. Ann. 161 (1965), 241-246.

[12] S. P. Redmond, The zero-divisor graph of a non-commutative ring, Commutative rings, 39-47, Nova Sci. Publ., Hauppauge, NY, 2002.

[13] - Structure in the zero-divisor graph of a noncommutative ring, Houston J. Math. 30 (2004), no. 2, 345-355

[14] T. Wu, On directed zero-divisor graphs of finite rings, Discrete Math. 296 (2005), no. $1,73-86$.

Department of Mathematics Education

Pusan National University

PUSAN 609-735, Korea

E-mail address: jchan@pusan.ac.kr 